PROCEEDINGS OF THE

AMERICAN MATHEMATICAL SOCIETY

Volume 138, Number 11, November 2010, Pages 3911-3921

S 0002-9939(2010)10297-6

Article electronically published on July 1, 2010

\title{
AN INVERSE PROBLEM FOR THE HEAT EQUATION
}

\author{
AMIN BOUMENIR AND VU KIM TUAN \\ (Communicated by Peter A. Clarkson)
}

\begin{abstract}
We prove that we can uniquely recover the coefficient of a one dimensional heat equation from a finite set of measurements and provide a constructive procedure for its recovery. The algorithm is based on the well known Gelfand-Levitan-Gasymov inverse spectral theory of Sturm-Liouville operators. By using a hot spot, as a first initial condition, we determine nearly all except maybe a finite number of spectral data. A counting procedure helps detect the number of missing data which is then unraveled by a finite number of measurements.
\end{abstract}

\section{INTRODUCTION}

Consider the one-dimensional heat process in a finite rod of the length, say $\pi$, where the heat source is proportional to the temperature distribution $u(x, t)$,

$$
\left\{\begin{array}{l}
u_{t}(x, t)=u_{x x}(x, t)-q(x) u(x, t), \quad 0 \leq x \leq \pi, t \geq 0, \\
u(0, t)-h u_{x}(0, t)=0 \\
u(\pi, t)+H u_{x}^{\prime}(\pi, t)=0 \\
u(x, 0)=f(x) .
\end{array}\right.
$$

We are concerned with the recovery of the coefficient $q$ from the measurements of $u(0, t)$, which is the heat released at one end of the rod. The constants $h$ and $H$ in the lateral boundary conditions correspond to the insulation parameters at both ends, and $1 / h$ is called the convective heat transfer coefficient. These parameters basically control the amount of heat released through the end points. To express the dependence of $u$ on the initial temperature $f$ and the convective heat transfer coefficient $h$, we sometimes use the notation $u=u^{f}$ and $u=u^{h}$ interchangeably when needed.

It is well known that by the maximum principle and variational arguments, $q$ is uniquely determined by the full lateral Dirichlet-to-Neumann map, $u^{f}(0, t) \rightarrow$ $u_{x}^{f}(0, t)$, that is, all possible lateral boundary conditions, [12, 13, 8, 5, 7]. In [18, the strategy is to introduce another family of source terms $\psi_{j}$, independent of the temperature distribution $u(x, t)$, and then solve the countable family of equations $u_{t}=u_{x x}(x, t)-q(x) u(x, t)+\psi_{j}(x)$ with Dirichlet boundary and initial conditions, i.e. $u(x, 0)=u(0, t)=u(\pi, t)=0$. It is shown that if the sequence $\left\{\psi_{j}\right\}_{j \geq 0}$ is complete in $L^{2}(0, \pi)$, then $q$ can be uniquely determined by the sequence

Received by the editors October 5, 2007.

2010 Mathematics Subject Classification. Primary 35R30, 34K29.

Key words and phrases. Heat equation, inverse spectral problem.

(C)2010 American Mathematical Society 
$\left\{\frac{\partial}{\partial x} u\left(0, t^{*}\right)\right\}$ when $q$ is small enough and $t^{*}$ is large enough. The use of the full Dirichlet-to-Neumann map is an idealized setting and is not applicable in real life.

We show that for any $q \in L^{1}(0, \pi)$ there exists $N$ such that $q$ is uniquely determined by a finite number of measurements $f_{i}(x) \rightarrow u^{f_{i}}(0, t), t \in(0, T), i=1, \ldots, N$, that are read for two different boundary conditions $h_{1} \neq h_{2}$.

Our approach is new since we neither add internal sources $\psi_{j}(x)$ nor use the full Dirichlet-to-Neumann map $u(0, t) \rightarrow u_{x}(0, t)$, but we base our recovery algorithm of $q$ only on a finite number of measurements $\left\{u^{f_{k}}(0, t)\right\}$.

To this end we use the classical method of separation of variables to describe how an inverse Sturm-Liouville problem can be used to solve an inverse problem for the heat equation. This is crucial in applied mathematics since some useful methods are available for the numerical solution of inverse Sturm-Liouville problems, 1, 9], but there are less numerical methods available for inverse problems involving PDEs.

The main issue is to extract a countable and complete set of eigenvalues associated with $q$ from a finite number of measurements $u^{f}(0, t)$. Fourier analysis then shows that the special initial condition, $f(x)=x^{\alpha}$, with $-1 / 2<\alpha<0$ guarantees the presence of all large eigenvalues in $u^{f}(0, t)$. The next key step in the algorithm uses a counting argument to determine the number of missing eigenvalues from the first measurement $u^{x^{\alpha}}(0, t)$. The algorithm finds those few missing eigenvalues from $u^{x^{k}}(0, t)$, where $k=0, \ldots, N$, in a finite number of steps. We also present several ways to extract eigenvalues from $u(0, t)$ or its samples $\left\{u\left(0, t_{n}\right)\right\}_{n \geq 0}$.

Once a complete set of spectral data has been obtained, we then repeat the same procedure with a different value of $h$ in (1) to get a second complete set of eigenvalues. Then $q$ can be uniquely recovered from the two sets of spectral data 14, 15.

Our main contribution here is to describe an algorithm on how to recover $q$ uniquely from a finite number of measurements, and so there is no need for the full Dirichlet-to-Neumann map. A future work is the numerical implementation of our algorithm, where one needs to overcome the ill-posedness and the usual scarcity of data in real world.

\section{Notation}

Before we start the inverse problem we first work out the direct problem to characterize the solution $u$ in (11). Let $\lambda_{n}$ and $\varphi_{n}$ be the eigenvalues and associated eigenfunctions of the Sturm-Liouville problem

$$
\left\{\begin{array}{l}
-\varphi_{n}^{\prime \prime}(x)+q(x) \varphi_{n}(x)=\lambda_{n} \varphi_{n}(x), \quad 0 \leq x \leq \pi \\
\varphi_{n}^{\prime}(0)-h_{1} \varphi_{n}(0)=0 \\
\varphi_{n}^{\prime}(\pi)+H \varphi_{n}(\pi)=0
\end{array}\right.
$$

Given a potential $q$, the general solution $u^{h_{1}}=u$ of (1) can be obtained by a separation of variables

$$
u(x, t)=\sum_{n \geq 0} c_{n} e^{-\lambda_{n} t} \varphi_{n}(x),
$$

where $\varphi_{n}$ are normalized by $\varphi_{n}(0)=1$. Obviously, each $c_{n}$ depends on the initial condition only,

$$
u(x, 0)=\sum_{n \geq 0} c_{n} \varphi_{n}(x) \text { and } u(0, t)=\sum_{n \geq 0} c_{n} e^{-\lambda_{n} t},
$$


and $u(x, 0)=f(x) \in L^{2}(0, \pi)$ if and only if

$$
\sum_{n \geq 0}\left|c_{n}\right|^{2}<\infty
$$

Observe that the last representation in (4) is unique, which means that given the function $u(0, t)$ one can recover the sequence of eigenvalues $\lambda_{n}$ whenever $c_{n} \neq 0$. In Section 3 we explain how to extract these eigenvalues $\lambda_{n}$, when $c_{n} \neq 0$. The novelty here is the condition that guarantees $c_{n} \neq 0$ and for such $n$ that $c_{n}=0$ we are missing the corresponding eigenvalue $\lambda_{n}$ (and with it the knowledge of the $n^{\text {th }}$ generalized Fourier mode $\varphi_{n}(x)$ of $q(x)$ ). In Sections 4 and 5 we propose a way around this. First, an input function of the form $f(x)=x^{\alpha}$ for $-\frac{1}{2}<\alpha<0$ is used. It is shown that this prevents $c_{n}=0$ for all $n>N$ for $N$ sufficiently large. Although there is no way to explicitly determine the value of $N$, as it depends only on the unknown $q$, we can estimate $N$ by using the counting principle. Next, the lower eigenvalues are sought by successive inputs of the family $f_{k}(x)=x^{k}$ for $k=0,1, \ldots, N-1$. Thus we can determine the full set of eigenvalues $\left\{\lambda_{n}\right\}_{n \geq 0}$, associated with $q, h_{1}, H$. To proceed to the next step assume that the complete set of eigenvalues $\left\{\lambda_{n}\right\}_{n \geq 0}$ has been recovered. Recall that to determine a potential $q$ we need two sets of eigenvalues, which is one of the main results of the Gelfand-LevitanGasymov theory [15, 14, 11, 16, 17. Assume that $q^{\prime} \in L^{1}(0, \pi)$ and $q(x), h, H \in \mathbb{R}$. Then the regular Sturm-Liouville operator $L$, defined by

$$
\left\{\begin{array}{l}
L(y)(x):=-y^{\prime \prime}(x)+q(x) y(x), \quad 0 \leq x \leq \pi, \\
y(0)-h y^{\prime}(0)=0, \quad y(\pi)+H y^{\prime}(\pi)=0
\end{array}\right.
$$

has an infinite sequence of eigenvalues $\left\{\lambda_{n}\right\}_{n>0}$, where $\lambda_{n} \uparrow \infty$. By choosing two different constants $h_{1} \neq h_{2}$, for the boundary condition at $x=0$, while keeping the same potential $q$ and constant $H$, we obtain two interlacing sequences of eigenvalues

$$
\lambda_{0}<\mu_{0}<\lambda_{1}<\mu_{1}<\ldots<\lambda_{n}<\mu_{n}<\ldots
$$

where

(7)

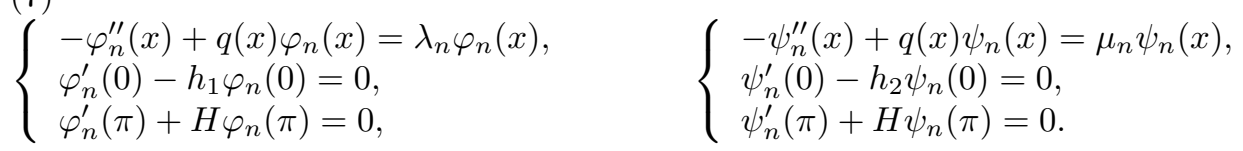

For convenience the eigenfunctions are normalized by $\varphi_{n}(0)=\psi_{n}(0)=1$. We now recall the Gasymov-Levitan Theorem [15], [14, Theorem 3.3.1], which uses the following properties of the eigenvalues of problems (7):

$$
\begin{gathered}
\lambda_{0}<\mu_{0}<\lambda_{1}<\mu_{1}<\ldots<\lambda_{n}<\mu_{n}<\ldots, \\
\sqrt{\lambda_{n}}=n+\frac{a_{0}}{n}+\frac{a_{1}}{n^{3}}+O\left(\frac{1}{n^{4}}\right) \text { and } \sqrt{\mu_{n}}=n+\frac{a_{0}^{\prime}}{n}+\frac{a_{1}^{\prime}}{n^{3}}+O\left(\frac{1}{n^{4}}\right), \\
\text { with } a_{0} \neq a_{0}^{\prime} .
\end{gathered}
$$

Theorem 1 (Gasymov-Levitan). Let two sequences of real numbers, $\left\{\lambda_{n}\right\}_{n \geq 0}$ and $\left\{\mu_{n}\right\}_{n \geq 0}$, satisfy (8) and (9). Then there exist a unique function $q$, constants $h_{1}$, $h_{2}$ and $H$ such that $q^{\prime} \in L^{1}(0, \pi)$, and the sequences $\left\{\lambda_{n}\right\}_{n \geq 0}$ and $\left\{\mu_{n}\right\}_{n \geq 0}$ are the eigenvalues of the Sturm-Liouville problems in (7). 
Obviously given $q$, and changing $h_{1}$ to $h_{2}$, yields a similar solution

$$
u^{h_{2}}(x, t)=\sum_{n \geq 0} d_{n} e^{-\mu_{n} t} \psi_{n}(x)
$$

so that

$$
u^{h_{2}}(x, 0)=\sum_{n \geq 0} d_{n} \psi_{n}(x) \text { and } u^{h_{2}}(0, t)=\sum_{n \geq 0} d_{n} e^{-\mu_{n} t},
$$

from which we can determine a second set of eigenvalues $\left\{\mu_{n}\right\}_{n \geq 0}$ associated with $q, h_{2}, H$.

Algorithms that recover $q$ from two spectra $\left\{\lambda_{n}\right\}_{n \geq 0}$ and $\left\{\mu_{n}\right\}_{n \geq 0}$ are given in [1, 9.

Observe that only the eigenvalues $\lambda_{n}$ and $\mu_{n}$, which are buried in $u^{h_{1}}(0, t)$ and $u^{h_{2}}(0, t)$, respectively, are required for the recovery of $q$. Thus the knowledge of $c_{n}$, or equivalently $f(x)$, is not used. This feature makes the algorithm different from the usual Dirichlet-to-Neumann map approach.

\section{EXtracting EIGENVALUES}

In practice the problem of recovering the frequencies of a function from its values is known as spectral estimation, and their extraction depends on the available data $u(0, t)$. Thus assume that $g$ is a function known to have the following representation:

$$
g(t)=\sum_{n \geq 0} c_{n} e^{-\lambda_{n} t}, \quad \text { where } \sum_{n \geq 0}\left|c_{n}\right|^{2}<\infty, \quad c_{n} \neq 0,
$$

$\lambda_{n} \uparrow \infty$ and $c_{n} \in \mathbb{R}$. We now outline several ways to recover its "frequencies" $\lambda_{n}$. Recall that when the frequencies are purely complex, sampling the function at $t_{k}$ leads to a linear system that can be solved by methods of linear algebra 21. Also, classical techniques from almost periodic functions recover the Bohr spectrum defined by supp $\sigma=\left\{i \lambda_{n}\right\}_{n \geq 0}$, where $\sigma(\lambda):=\lim _{T \rightarrow \infty} \frac{1}{2 T} \int_{-T}^{T} \exp (i \lambda t) g(i t) d t$. However, since in our case the frequencies are real numbers, we give different algorithms.

3.1. Laplace transform. If $u(0,)=$.$g is known for all t>0$, then we can use the Laplace transform $\mathcal{L}(g)(s)=\int_{0}^{\infty} e^{-s t} g(t) d t$.

Proposition 1. Assume that a function $g$ has the representation (11) for all $t>0$; then the sequence $\left\{-\lambda_{n}\right\}_{n \geq 0}$ corresponds to the zeros of $1 / \mathcal{L}(g)(s)$.

Proof. We have that

$$
\mathcal{L}(g)(s)=\mathcal{L}\left(\sum_{n \geq 0} c_{n} e^{-\lambda_{n} t}\right)=\sum_{n \geq 0} \frac{c_{n}}{s+\lambda_{n}},
$$

the series converges to an analytic function on $\Re(s)>-\lambda_{0}$ and is meromorphic over the complex plane. Observe that since the eigenvalues $\left\{-\lambda_{n}\right\}_{n \geq 0}$ coincide with the poles of the meromorphic function $\mathcal{L}(g)(s)$, they are the zeros of $\frac{1}{\mathcal{L}(g)(s)}$. Standard root-finding methods can be used to detect $\lambda_{n}$. 
3.2. The method of limits. We now assume that only the sequence of values $\{g(k)\}_{k \geq 0}$ is known. The argument can easily be extended to $\left\{g\left(t_{k}\right)\right\}_{k \geq 0}$, where $t_{k}$ is an arbitrary increasing sequence approaching infinity.

Proposition 2. Assume that a function $g$ has the representation (11). Then we can recover the sequence $\left\{\lambda_{n}\right\}_{n \geq 0}$ from $\{g(k)\}_{k \geq 0}$ by taking $\lim _{k \rightarrow \infty} \frac{g(k)}{g(k+1)}$.

Proof. Since the eigenvalues are increasing, $\lambda_{0}<\lambda_{1}<\ldots<\lambda_{n}<\ldots$. Then for large $k$, we have $g(k) \sim c_{0} e^{-\lambda_{0} k}$, and so the limit

$$
\lim _{k \rightarrow \infty} \frac{g(k)}{g(k+1)}=\lim _{k \rightarrow \infty} \frac{e^{-\lambda_{0} k}}{e^{-\lambda_{0}(k+1)}}=e^{\lambda_{0}} \Rightarrow \lambda_{0}=\lim _{k \rightarrow \infty} \ln \left(\frac{g(k)}{g(k+1)}\right) .
$$

We can compute $c_{0}$ by another limit,

$$
\lim _{k \rightarrow \infty} g(k) e^{\lambda_{0} k}=c_{0},
$$

since $\lambda_{0}-\lambda_{n}<0, n \geq 1$. By removing the first term $c_{0} e^{-\lambda_{0} t}$ from the series defining the original $g$, we obtain a new function,

$$
g_{1}(t)=g(t)-c_{0} e^{-\lambda_{0} t}=\sum_{n \geq 1} c_{n} e^{-\lambda_{n} t} .
$$

To recover the next pair $\left(\lambda_{1}, c_{1}\right)$ we only need to repeat steps (13) and (14) to the function $g_{1}$. By doing so we can eventually determine all the sequence $\left\{\lambda_{n}\right\}_{n \geq 0}$.

3.3. Boundary control methods. Assume that we can choose $u(x, 0)=f(x)$, so that $u(0, t)=g(t)=\sum_{n=0}^{N} c_{n} e^{-\lambda_{n} t}$ is now a finite sum of exponentials, or we truncate the series in $g$ to get only a finite sum. In this case the Boundary Control method applies [5, 6, 8. If we denote by $A$ and $B$ the $N \times N$ matrices defined by $A_{i j}=g(i+j-1)$ and $B_{i j}=g(i+j-2)$, then it is shown that $\lambda_{n}$ are precisely the eigenvalues of the pencil

$$
A-\lambda B .
$$

The same result (15) is also obtained through a different approach which takes into account noise; see [20, 21].

The problem considered here is idealized; namely, $u(0, t)$ is assumed to be known for all $t>0$ or, at least, for a sequence $t_{k}$ approaching infinity. In a practical situation, measurements must be taken over a finite time. This leads to the classical challenge in the practical numerical solution of inverse Sturm-Liouville problems, namely, how to obtain the best possible information when only finitely many eigenvalues are known with some level of accuracy. We will not go into detail here, but refer the reader to [19, where the problem of dealing with limited data in the inverse Sturm-Liouville problem is considered. Further related references are given (with some discussion) in [1, 2, 10.

\section{The HOt SPOT Method}

Now that we know how to extract the $\lambda_{n}$ from the measurement $u(0, t)$, we face the question of their completeness. Thus given any $f \in L^{2}(0, \pi)$, we have $f(x)=\sum_{n \geq 0} c_{n} \varphi_{n}(x)$, and to retrieve all eigenvalues $\lambda_{n}$ from (11) we need to ensure that $c_{n} \neq 0$ for all $n \geq 0$. The main difficulty here is that since the system $\varphi_{n}$ is unknown, the condition $c_{n} \neq 0$ cannot be checked out. The key idea is to use a special initial condition, with a hot spot, i.e. $\lim _{x \rightarrow 0^{+}} f(x)=\infty$, to ensure 
that its expansion contains nearly all eigenfunctions. We start with the following proposition.

Proposition 3 (Hot spot). Let $q \in L^{1}(0, \pi), \varphi_{n}$ be defined by (7) and $f(x)=x^{\alpha}$, where $\alpha \in(-1 / 2,0)$. Then there is $N>0$ such that $c_{n} \neq 0$ for $n \geq N$.

Proof. We first recall the existence of a kernel $K$ whose first derivatives are locally integrable such that

$$
\varphi_{n}(x)=\cos \left(x \sqrt{\lambda_{n}}\right)+\int_{0}^{x} K(x, t) \cos \left(t \sqrt{\lambda_{n}}\right) d t .
$$

In this case

$$
\begin{aligned}
\left\|\varphi_{n}\right\|^{2} \quad c_{n} & =\int_{0}^{\pi} f(x) \varphi_{n}(x) d x \\
& =\int_{0}^{\pi} f(x) \cos \left(x \sqrt{\lambda_{n}}\right) d x+\int_{0}^{\pi} \int_{x}^{\pi} K(t, x) f(t) d t \cos \left(x \sqrt{\lambda_{n}}\right) d x .
\end{aligned}
$$

We now use the asymptotic of $\lambda_{n}$ in (9) to deduce the asymptotics of the integrals in (16). By the mean value theorem,

$$
\cos \left(x \sqrt{\lambda_{n}}\right)=\cos (x n)+\frac{x}{n}(a+o(1)) \sin \left(x \xi_{n}\right),
$$

where $\xi_{n}$ is between $\sqrt{\lambda_{n}}$ and $n$. Then

$$
\int_{0}^{\pi} f(x) \frac{x}{n}(a+o(1)) \sin \left(x \xi_{n}\right) d x=O\left(\frac{1}{n}\right),
$$

while if $f(x)=x^{\alpha}$, then

$$
\int_{0}^{\pi} f(x) \cos (x n) d x=\int_{0}^{\pi} x^{\alpha} \cos (x n) d x=n^{-\alpha-1} \int_{0}^{\pi}(x n)^{\alpha} \cos (x n) d(x n) .
$$

Since $\alpha \in(-1 / 2,0)$, the cosine integral is convergent:

$$
\begin{aligned}
\lim _{n \rightarrow \infty} \int_{0}^{\pi}(x n)^{\alpha} \cos (x n) d(x n) & =\lim _{n \rightarrow \infty} \int_{0}^{n \pi} \xi^{\alpha} \cos (\xi) d \xi=\int_{0}^{\infty} \xi^{\alpha} \cos (\xi) d \xi \\
& =c(\alpha)=\sqrt{\pi} 2^{\alpha} \frac{\Gamma((\alpha+1) / 2)}{\Gamma(-\alpha / 2)}
\end{aligned}
$$

and so

$$
\int_{0}^{\pi} f(x) \cos (x n) d x \approx \frac{c(\alpha)}{n^{\alpha+1}} .
$$

Thus we have proved that when $\alpha \in(-1 / 2,0)$, we have

$$
\int_{0}^{\pi} x^{\alpha} \cos \left(x \sqrt{\lambda_{n}}\right) d x=\frac{c(\alpha)}{n^{\alpha+1}}+O\left(\frac{1}{n}\right) .
$$


In the same way, we can split the second integral:

$$
\begin{aligned}
\int_{0}^{\pi} \int_{x}^{\pi} K(t, x) f(t) d t \cos \left(x \sqrt{\lambda_{n}}\right) d x & =\int_{0}^{\pi} \int_{x}^{\pi} K(t, x) f(t) d t \cos (x n) d x \\
& +\frac{(a+o(1))}{n} \int_{0}^{\pi} \int_{x}^{\pi} K(t, x) f(t) d t x \sin \left(x \xi_{n}\right) d x \\
& =\int_{0}^{\pi} \int_{x}^{\pi} K(t, x) f(t) d t \cos (x n) d x+O\left(\frac{1}{n}\right) .
\end{aligned}
$$

To estimate the first integral we use the fact that $\int_{x}^{\pi} K(t, x) t^{\alpha} d t$ is absolutely continuous. Indeed its derivative is

$$
\frac{d}{d x} \int_{x}^{\pi} K(t, x) t^{\alpha} d t=-K(x, x) x^{\alpha}+\int_{x}^{\pi} K_{x}(t, x) t^{\alpha} d t
$$

where $K(x, x) x^{\alpha} \in L(0, \pi)$. Since $K$ is a continuous function and

$$
\left|\int_{x}^{\pi} K_{x}(t, x) t^{\alpha} d t\right| \leq x^{\alpha} \int_{x}^{\pi}\left|K_{x}(t, x)\right| d t \leq x^{\alpha} \sup _{0 \leq x \leq \pi} \int_{x}^{\pi}\left|K_{x}(t, x)\right| d t,
$$

we deduce that $\int_{x}^{\pi} K_{x}(t, x) t^{\alpha} d t=O\left(x^{\alpha}\right)$, and so $\int_{x}^{\pi} K_{x}(t, x) t^{\alpha} d t \in L(0, \pi)$ when $\alpha \in(-1 / 2,0)$. Thus $\int_{x}^{\pi} K(t, x) t^{\alpha} d t$ is absolutely continuous and its Fourier coefficients satisfy

$$
\int_{0}^{\pi} \int_{x}^{\pi} K(t, x) f(t) d t \cos (x n) d x=o\left(\frac{1}{n}\right) \text { as } n \rightarrow \infty .
$$

In this case, combining (17) and (18) yields

$$
\int_{0}^{\pi} \int_{x}^{\pi} K(t, x) f(t) d t \cos \left(x \sqrt{\lambda_{n}}\right) d x=o\left(\frac{1}{n}\right)+O\left(\frac{1}{n}\right)=O\left(\frac{1}{n}\right) .
$$

and we finally obtain that

$$
\int_{0}^{\pi} x^{\alpha} \varphi_{n}(x) d x=\frac{c(\alpha)}{n^{\alpha+1}}+O\left(\frac{1}{n}\right) .
$$

Also, since $-1 / 2<\alpha<0$ it follows that

$$
c_{n} n^{\alpha+1}\left\|\varphi_{n}\right\|^{2} \rightarrow c(\alpha) \neq 0,
$$

and the result follows.

We have shown that the choice $f(x)=x^{\alpha},-1 / 2<\alpha<0$, guarantees the existence of $N$ such that $c_{n} \neq 0$ for $n \geq N$. The actual value $N$ follows from the constant in $O$. Section 3 gave us the method for extracting $\lambda_{n}$ from $u^{x^{\alpha}}(0, t)$ for $n \geq N$. In the next section we show how to find the number of missing eigenvalues and choose $f$ so that the first $c_{n}, n=0, \ldots, N-1$, are also nonzero.

\section{Recovering the Missing EIGENVALUes}

To proceed further we need to find out the number of missing eigenvalues and then recover them before we can use the Gasymov-Levitan theorem. Although we know the existence of $N$ from the previous section, its actual value remains unknown and cannot be utilized directly in the counting argument. Instead, let $M$ be the number starting from which all recovered eigenvalues larger than $(M-1 / 2)^{2}$ fall in enclosures of the form $\left((k-1 / 2)^{2},(k+1 / 2)^{2}\right)$, where $k \geq M$, and more importantly 
each of these intervals contains exactly one recovered eigenvalue. The existence of such a value $M$ comes from (9) and Proposition 3, which says that after a certain $N$, no eigenvalue is missing. Therefore we can tentatively rank the eigenvalue contained in $\left((k-1 / 2)^{2},(k+1 / 2)^{2}\right), k \geq M$, as $\lambda_{k}$, and this ranking is true for $k \geq P=\max (N, M)$.

Now suppose there are only $L$ eigenvalues recovered on $\left(-\infty, \lambda_{M}\right)$. Since $\left[\lambda_{M}, \lambda_{P}\right)$ contains $P-M$ eigenvalues $\left\{\lambda_{M}, \ldots, \lambda_{P-1}\right\}$, the actual number of recovered eigenvalues in $\left(-\infty, \lambda_{P}\right)$ is $L+(P-M)$. From $\lambda_{P}$ and up the ranking is true, and therefore there must be $P$ eigenvalues before $\lambda_{P}$. Hence, $M-L$ eigenvalues are missing. Thus by knowing $M$, we also know the number of missing eigenvalues.

For example, starting from $k=100$, each of the intervals $\left((k-1 / 2)^{2}\right.$, $\left.(k+1 / 2)^{2}\right)$ contains exactly one recovered eigenvalue, and none falls outside. Then the eigenvalue inside $\left((k-1 / 2)^{2},(k+1 / 2)^{2}\right), k \geq 100$, can be ranked tentatively as $\lambda_{k}$. If by counting we have found only 80 eigenvalues less than $\lambda_{100}$, it means that we are missing only 20 eigenvalues. The difficulty in ranking here is that the missing eigenvalues are not necessarily below $\lambda_{100}$. That is why it is important to first recover all the missing eigenvalues; only after that can the ranking be finalized.

To this end we propose a way to dig out all the missing eigenvalues in $\left\{\lambda_{0}, \ldots\right.$, $\left.\lambda_{P-1}\right\}$ by choosing appropriate initial conditions to ensure that the missing eigenvalues are necessarily embedded in the response $u^{f}(0, t)$. In other words we need to find initial conditions that would guarantee $c_{n} \neq 0$ for $n=0, \ldots, P-1$. We start with the following observation.

Proposition 4. Given $\varphi_{j}$, the coefficients $c_{j}(k)=\int_{0}^{\pi} x^{k} \varphi_{j}(x) d x \neq 0$ for at least one $k=0, \ldots, j$.

Proof. Let us assume that

$$
c_{j}(k)=0 \text { for all } k=0, \ldots, j .
$$

Recall that the $j^{\text {th }}$ eigenfunction of (7) has $j$ simple zeroes, say $a_{j k}$, and so $\varphi_{j}\left(a_{j k}\right)$ $=0$ for $k=0, \ldots, j$. Given the zeros $a_{j k}$ we can, by interpolation, construct the polynomial

$$
P_{j}(x)=\varepsilon_{j} \prod_{k=0}^{j}\left(x-a_{j k}\right)=\sum_{k=0}^{j} b_{k} x^{k}
$$

where $\varepsilon_{j}$ is chosen so that $P_{j}(0)=1$. Since $P_{j}$ and $\varphi_{j}$ have similar zeros and both satisfy $P_{j}(0)=\varphi_{j}(0)=1$, they must have the same sign for all $x \in[0, \pi]$; that is,

$$
P_{j}(x) \varphi_{j}(x)>0 \text { if } x \neq a_{j k} .
$$

Therefore we conclude that

$$
\int_{0}^{\pi} P_{j}(x) \varphi_{j}(x) d x>0
$$

and at the same time by (19) and (20) we have

$$
\int_{0}^{\pi} P_{j}(x) \varphi_{j}(x) d x=\sum_{k=0}^{j} b_{k} \int_{0}^{\pi} x^{k} \varphi_{j}(x) d x=\sum_{k=0}^{j} b_{k} c_{j}(k)=0,
$$

which contradicts (21). 
The above result says that the family $\left\{u^{x^{k}}(0, t)\right\}_{k=0}^{P-1}$ will deliver the first $P$ eigenvalues, but unfortunately the number $P$ is unknown. Nevertheless, we can still use the knowledge of the number of missing eigenvalues, i.e. $M-L$, as a stopping rule.

Thus we start by reading $u^{x^{0}}(0, t)$ to find missing eigenvalues. If there are still some missing eigenvalues, we go to the next $u^{x^{1}}(0, t)$, and so on until we have recovered all the $M-L$ missing eigenvalues. Proposition 4 guarantees that this process stops at some point (at most after looking at $u^{x^{P-1}}(0, t)$ ). After that we can finally rearrange and update the previous ranking.

\section{The Algorithm}

We now summarize the algorithm, which reconstructs $q$ in finite steps.

Step 1. Fix the value $h_{1}$ in (11) and start with a hot spot $u(x, 0)=x^{\alpha}$ where $\alpha \in(-1 / 2,0)$. Read the temperature $u(0, t)$ and then extract the frequencies $\lambda_{k_{j}}$. Proposition 3 guarantees that all $\lambda_{k}$ are recovered after a certain $\lambda_{N}$.

Step 2. Find the smallest $M$ such that starting from $M$ in each of the intervals $\left((k-1 / 2)^{2},(k+1 / 2)^{2}\right), k \geq M$, there is exactly one eigenvalue recovered in Step 1 , and none falls outside. Tentatively rank it as $\lambda_{k}$.

Step 3. Count the number of eigenvalues obtained from Step 1 in the interval $\left(-\infty, \lambda_{M}\right)$, say $L$. It will be less than or equal to $M$. Then $M-L$ is the number of eigenvalues not recovered in Step 1. All the missing $M-L$ eigenvalues can now be recovered by trying $\left\{u^{x^{j}}(0, t)\right\}, j=0,1, \ldots$, step by step until new $M-L$ eigenvalues are discovered.

Step 4. Change the boundary condition from $h_{1}$ to $h_{2}$ and repeat Steps $1-3$ to find another set of eigenvalues $\left\{\mu_{k}\right\}$.

Step 5. Knowing the sequences $\left\{\lambda_{n}\right\}$ and $\left\{\mu_{k}\right\}$, use an algorithm and code that would recover $q$ from two spectra; see [1, 2, 9, 14, 16].

\section{THE UNIQUENESS}

Now we show that the measurements determine $q$ uniquely. More precisely, we have

Theorem 2. Let $T>0,-\frac{1}{2}<\alpha<0$, and $h_{1} \neq h_{2}$. For any $q \in L^{1}(0, \pi)$ there exists $N$ such that $q$ is uniquely determined by a finite number of measurements $u^{x^{\alpha}}(0, t), u^{x^{0}}(0, t), u^{x^{1}}(0, t), \ldots, u^{x^{N}}(0, t), t \in(0, T)$, that are read for two different boundary conditions $h=h_{1}$ and $h=h_{2}$.

Proof. Suppose for any $N>0$ two potentials $q, \tilde{q} \in L^{1}(0, \pi)$ give the same measurements,

$$
u^{x^{\alpha}}(0, t), u^{x^{0}}(0, t), u^{x^{1}}(0, t), \ldots, u^{x^{N}}(0, t), \quad t \in(0, T),
$$

for $h=h_{1}$. Let $\left\{\lambda_{n}\right\}$ and $\left\{\varphi_{n}(x)\right\}$ be the set of eigenvalues, numbered in increasing order, and the set of normalized eigenfunctions $\left(\varphi_{n}(0)=1\right)$ associated with $q$. Similarly, let $\left\{\tilde{\lambda}_{n}\right\}$ and $\left\{\tilde{\varphi}_{n}(x)\right\}$ be the set of eigenvalues and the set of normalized eigenfunctions associated with $\tilde{q}$. Thus, any $f \in L^{2}(0, \pi)$ can be expanded into two Fourier series,

$$
f(x)=\sum_{n \geq 0} c_{n} \varphi_{n}(x)=\sum_{n \geq 0} \tilde{c}_{n} \tilde{\varphi}_{n}(x) .
$$


The potential $q$ yields the heat distribution

$$
u(x, t)=\sum_{n \geq 0} c_{n} e^{-\lambda_{n} t} \varphi_{n}(x),
$$

with the heat released at the end point $x=0$ :

$$
u(0, t)=\sum_{n \geq 0} c_{n} e^{-\lambda_{n} t} .
$$

The potential $\tilde{q}$ yields the heat distribution

$$
\tilde{u}(x, t)=\sum_{n \geq 0} \tilde{c}_{n} e^{-\tilde{\lambda}_{n} t} \tilde{\varphi}_{n}(x),
$$

with the heat released at the end point $x=0$ :

$$
\tilde{u}(0, t)=\sum_{n \geq 0} \tilde{c}_{n} e^{-\tilde{\lambda}_{n} t} .
$$

Since $u(0, t)$ is analytic in $t>0$, the measurement of $u(0, t)$ on any finite time interval $(0, T)$ is sufficient to recover $u(0, t)$ uniquely for all $t>0$. Thus, $q$ and $\tilde{q}$ give the same measurements,

$$
u^{x^{\alpha}}(0, t), u^{x^{0}}(0, t), u^{x^{1}}(0, t), \ldots, u^{x^{N}}(0, t), \quad t \in(0, \infty),
$$

for $h=h_{1}$. In other words,

$$
\sum_{n \geq 0} c_{n} e^{-\lambda_{n} t}=\sum_{n \geq 0} \tilde{c}_{n} e^{-\tilde{\lambda}_{n} t}, \quad t>0 .
$$

An analysis of Proposition 2 tells us that (22) holds if and only if for any $n$ with $c_{n} \neq 0$ there exists a unique $k$ such that $\lambda_{n}=\tilde{\lambda}_{k}$ and $c_{n}=\tilde{c}_{k}$, and conversely, for any $k$ with $\tilde{c}_{k} \neq 0$ there exists unique $n$ such that $\lambda_{n}=\tilde{\lambda}_{k}$ and $c_{n}=\tilde{c}_{k}$. So, the set $\left\{\lambda_{n} \mid c_{n} \neq 0\right\}$ and the set $\left\{\tilde{\lambda}_{n} \mid \tilde{c}_{n} \neq 0\right\}$ are the same. But the set $\left\{\lambda_{n} \mid c_{n}^{f} \neq 0\right.$ for at least one $f$ from $x^{\alpha}$, and $\left.x^{j}, j=0, \ldots, N\right\}$ coincides with the set of eigenvalues $\left\{\lambda_{n}\right\}$, and the set $\left\{\tilde{\lambda}_{n} \mid \tilde{c}_{n}^{f} \neq 0\right.$ for at least one $f$ from $x^{\alpha}$, and $\left.x^{j}, j=0, \ldots, N\right\}$ coincides with the set of eigenvalues $\left\{\tilde{\lambda}_{n}\right\}$. Thus, $\left\{\lambda_{n}\right\}=\left\{\tilde{\lambda}_{n}\right\}$. Similarly, with another boundary condition $h=h_{2}$ we can show that the two new sets of eigenvalues $\left\{\mu_{k}\right\}$ and $\left\{\tilde{\mu}_{k}\right\}$, associated with potentials $q$ and $\tilde{q}$ and $h=h_{2}$, are the same. But Theorem 1 says that the potential is uniquely determined by two sets of eigenvalues. Thus $q=\tilde{q}$ and the theorem is proved.

\section{REFERENCES}

[1] A.L. Andrew, Computing Sturm-Liouville potentials from two spectra. Inverse Problems 22 (2006), no. 6, pp. 2069-2081. MR2277530(2007i:34013)

[2] A.L. Andrew, Numerov's method for inverse Sturm-Liouville problems. Inverse Problems 21 (2005), no. 1, pp. 223-238. MR2146173 (2006d:65076)

[3] S.A. Avdonin and S.A. Ivanov, Riesz bases of exponentials and divided differences (in Russian). Algebra i Analiz 13 (2001), no. 3, pp. 1-17; translation in St. Petersburg Math. J. 13 (2002), no. 3, pp. 339-351. MR1850184 (2002g:42009)

[4] S.A. Avdonin and S.A. Ivanov, Families of Exponentials. The Method of Moments in Controllability Problems for Distributed Parameter Systems. Cambridge University Press, Cambridge, 1995. MR1366650 (97b:93002)

[5] S.A. Avdonin, M.I. Belishev, and Yu. Rozhkov, The BC-method in the inverse problem for the heat equation. J. Inv. Ill-Posed Probl. 5 (1997), pp. 309-322. MR.1473633 (98f:80002) 
[6] S.A. Avdonin and M.I. Belishev, Boundary control and dynamical inverse problem for nonselfadjoint Sturm-Liouville operator. Control and Cybernetics 25 (1996), pp. 429-440. MR.1408711 (97k:93032)

[7] S.A. Avdonin, S. Lenhart, and V. Protopopescu, Determining the potential in the Schrödinger equation from the Dirichlet to Neumann map by the boundary control method. Inverse problems: modeling and simulation. J. Inv. Ill-Posed Probl. 13 (2005), pp. 317-330. MR2188615 (2006m:35374)

[8] M.I. Belishev, Canonical model of a dynamical system with boundary control in inverse problem for the heat equation (in Russian). Algebra i Analiz 7 (1995), pp. 3-32; translation in St. Petersburg Math. J. 7 (1996), pp. 869-890. MR1381977(97a:93029)

[9] A. Boumenir, The recovery of analytic potentials. Inverse Problems 15 (1999), no. 6, pp. 1405-1423. MR.1733208 (2000j:34018)

[10] R.H. Fabiano, R. Knobel, and B.D. Lowe, A finite difference algorithm for an inverse SturmLiouville problem. IMA J. Appl. Math. 15 (1995), pp. 75-88. MR.1311338 (95j:65084)

[11] G. Freiling and V. Yurko, Inverse Sturm-Liouville Problems and Their Applications. Nova Science Publishers, Inc., Huntington, New York, 2001. MR.2094651 (2005f:34001)

[12] V. Isakov, On uniqueness in inverse problems for semilinear parabolic equations. Arch. Rational Mech. Anal. 124 (1993), no. 1, 1-12. MR.1233645 (94h:35263)

[13] V. Isakov, Inverse Problems for Partial Differential Equations, 2nd ed. Applied Mathematical Sciences, 127, Springer, New York, 2006. MR2193218 (2006h:35279)

[14] B.M. Levitan, Inverse Sturm-Liouville Problems. VNU Science Press, Utrecht, 1987. MR.933088 (89b:34001)

[15] B.M. Levitan and M.G. Gasymov, Determination of a differential equation by two of its spectra. Russ. Math. Surveys 19 (1964), pp. 1-62. MR0162996 (29:299)

[16] J.R. McLaughlin, Analytical methods for recovering coefficients in differential equations from spectral data. SIAM Rev. 28 (1986), pp. 53-72. MR828436 (87d:34034)

[17] J.R. McLaughlin, Solving Inverse Problems with Spectral Data. Surveys on Solution Methods for Inverse Problems, pp. 169-194, Springer, 2000. MR1766744 (2001f:35431)

[18] B.D. Lowe and W. Rundell, The determination of a coefficient in a parabolic equation from input sources. IMA J. Appl. Math. 52 (1994), no. 1, pp. 31-50. MR1270801 (95b:35229)

[19] B.D. Lowe, M. Pilant, and W. Rundell, The recovery of potentials from finite spectral data. SIAM J. Math. Anal. 23 (1992), pp. 482-504. MR1147873 (93f:34024)

[20] Y. Hua and T.K. Sarkar, Matrix pencil method for estimating parameters of exponentially damped/undamped sinusoids in noise. IEEE Transactions of Acoustics, Speech, and Signal Processing 38(5) (1990), pp. 814-824. MR1051029 (91b:93160)

[21] Y. Hua, A.B. Gershman, and Q. Cheng, High-Resolution and Robust Signal Processing. Marcel Dekker, New York-Basel, 2004.

[22] T.K. Sarkar, M.C. Wicks, M. Salazar-Palma, and R.J. Bonneau, Smart Antennas. John Wiley \& Sons, Hoboken, New Jersey, 2003.

Department of Mathematics, University of West Georgia, Carrollton, Georgia 30118

E-mail address: boumenir@westga.edu

Department of Mathematics, University of West Georgia, Carrollton, Georgia 30118

E-mail address: vu@westga.edu 